\title{
Modélisation numérique des ségrégations lors de la solidification d'alliages Ti-Al
}

\author{
Anne Noeppel ${ }^{1, a}$, Lä̈titia Jacoutot ${ }^{1}$, Mabel Medina $^{2}$, Alexandru Ciobanas ${ }^{1}$, \\ Florin Baltaretu ${ }^{3}$, Kader Zaidat ${ }^{1}$, Ana-Maria Bianchi ${ }^{3}$, Yves Fautrelle ${ }^{1}$ \\ ET JACQUELINE ETAY ${ }^{1}$ \\ 1 EPM/SIMAP/CNRS, Institut National Polytechnique de Grenoble, BP 95, 38402 Saint Martin d'Hères Cedex, France \\ 2 FCEIA, Universidad Nacional Rosario, Pelligrini 250, 2000 Rosario, Argentina \\ 3 UTC Bucharest, Bd. Pache Protopopescu 66, 021414 Bucharest, Romania
}

Reçu le 15 mars 2007, accepté le 7 juin 2007

\begin{abstract}
Résumé - La solidification des alliages binaires est problématique dans la mesure où de nombreuses hétérogénéités susceptibles de fragiliser le produit apparaissent. Ces hétérogénéités peuvent être à la fois de composition (appelées ségrégations) et de structures. Les ségrégations présentent, en outre, l'inconvénient d'apparaître aussi bien à l'échelle mésoscopique (freckles) qu'à l'échelle du lingot (macroségrégation). Il apparaît que la gravité joue un rôle prédominant dans la formation et le devenir des hétérogénéités observées. S'affranchir de cette composante peut être un des enjeux des nouveaux procédés de solidification. Par ailleurs, les alliages de titane-aluminium présentent une solidification péritectique : au cours de la solidification péritectique deux phases solides sont présentes dans le lingot. Un modèle numérique de solidification basé sur le moyennage statistique a été établi qui prend en compte la spécificité de ces alliages. Les calculs menés en condition de microgravité et de convection forcée montrent que la convection au voisinage du front de solidification influence la localisation des hétérogénéités.
\end{abstract}

Mots clés : Solidification / Ti-Al / modélisation numérique / ségrégation / péritectique

Abstract - Numerical modeling of segregations during the solidification of Ti-Al alloys. The solidification of binary alloys is problematic insofar as much heterogeneity likely to weaken the product appears. These heterogeneities can be at the same time of composition (called segregations) and of structures. The segregations present, moreover, the disadvantage of as well appearing on a mesoscopic scale (freckles) as on an ingot scale (macrosegregation). It appears that gravity plays a prevalent part in the formation and the evolution of heterogeneities. Moreover the titanium-aluminium alloys present peritectic solidification: during peritectic solidification two solid phases are present in the ingot. A numerical modelling of solidification based on the statistical average was established which takes into account the specificity of these alloys. The calculations carried out in condition of microgravity and forced convection show that the convection in the vicinity of the front of solidification influences the localization of heterogeneities.

Key words: Solidification / Ti-Al / numerical modelling / segregation / peritectic

\section{Introduction}

Dans le domaine aéronautique, un gain d'énergie considérable est attendu de l'accroissement du diamètre des aubes de turbine et de l'amélioration de leurs qualités mécaniques. Or ceci ne peut être atteint que si la solidification d'alliages de titane-aluminium est parfaitement maîtrisée. C'est l'un des objectifs du programme intégré de la communauté européenne IMPRESS (pour

\footnotetext{
a Auteur correspondant : anne.noeppel@hmg.inpg.fr
}

InterMetallic Processing in Relation to Earth and Space Solidification).

L'inconvénient majeur rencontré lors de la solidification des alliages binaires réside dans la présence d'hétérogénéités. Ces hétérogénéités sont en général de deux types; de composition (appelées « ségrégations ») et de structure. Ces hétérogénéités, inhérentes au processus de solidification, amenuisent, entre autres, les propriétés mécaniques et thermiques des alliages. Pour parvenir à maîtriser ce processus, les modèles numériques de 


\section{Nomenclature}

\begin{tabular}{|lll|}
\hline$C$ & concentration & $\% \mathrm{wt}$ \\
$T$ & température & $\mathrm{K}$ \\
$F$ & force volumique & $\mathrm{N} \cdot \mathrm{m}^{-3}$ \\
$G$ & gradient thermique & $\mathrm{K} \cdot \mathrm{m}^{-1}$ \\
$L$ & chaleur latente de fusion & $\mathrm{J} \cdot \mathrm{kg}^{-1}$ \\
$r$ & rayon & $\mathrm{m}$ \\
$R_{\text {cylindre }}$ & rayon du cylindre & $\mathrm{m}$ \\
$t$ & temps & $\mathrm{s}$ \\
$y$ & abscisse & $\mathrm{m}$ \\
& $\quad$ Lettres grecques & \\
$\alpha$ & phase solide secondaire & \\
$\beta$ & phase solide primaire & \\
$\delta$ & épaisseur de peau & $\mathrm{m}$ \\
$\lambda$ & conductivité thermique & $\mathrm{W} \cdot \mathrm{m}^{-1} \cdot \mathrm{K}^{-1}$ \\
$\rho$ & densité & $\mathrm{kg} \cdot \mathrm{m}^{-3}$ \\
& \multicolumn{2}{c}{ Indices } \\
$-p$ & péritectique & \\
$-L$ & liquidus & \\
\hline
\end{tabular}

solidification sont d'importance capitale pour comprendre la formation et l'évolution de ces hétérogénéités.

Dans cet article, nous présentons d'abord les particularités des alliages métalliques que nous considérons. Nous rappellerons ensuite brièvement le modèle employé. Enfin nous présenterons des résultats dans un cas de microgravité et dans un cas de convection forcée.

\section{Particularités des alliages de titane-aluminium}

\subsection{Ségrégation}

Dans les alliages binaires, les deux éléments en présence peuvent présenter des propriétés de solubilité telles que le soluté s'incorpore difficilement dans une des deux phases (la phase solide en général). Ainsi au cours de la solidification, le solide « rejette » le soluté en avant du front de solidification, ce qui a pour conséquence d'augmenter la concentration en soluté du bain liquide. Le lingot n'étant pas infini, une accumulation en soluté dans le bain liquide existe. Ainsi, la partie du lingot solidifiée dans les premiers temps aura une composition plus pauvre en soluté que la partie solidifiée plus tardivement. Ces différences de composition forment les ségrégations. Les ségrégations, phénomène intrinsèque de la solidification des alliages métalliques, sont gênantes dans la mesure où elles sont source d'hétérogénéités de composition des lingots ainsi obtenus. Les ségrégations sont particulièrement influencées par les phénomènes convectifs [1]. Deux possibilité s'offrent alors : soit on supprime la convection en se plaçant dans des conditions de microgravité, soit on effectue un contrôle de la convection (par procédé électromagnétique), ce qui permettrait un contrôle des ségrégations.

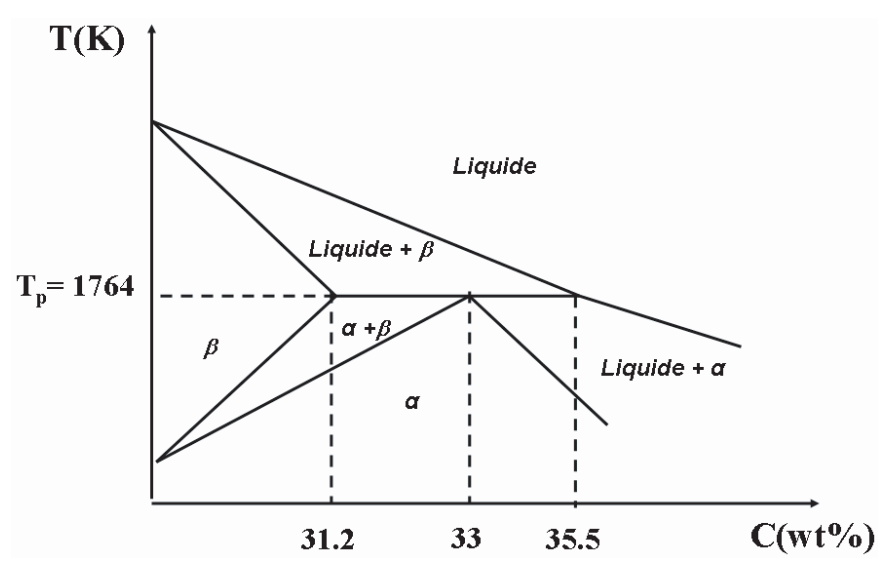

Fig. 1. Diagramme de phase d'un alliage péritectique. A $T=$ $T_{\mathrm{p}}$ : liquide + phase primaire $(\beta) \rightarrow$ phase secondaire $(\alpha)$.

\subsection{Solidification péritectique}

Pour réaliser les aubes de turbine, il est envisagé de travailler sur des alliages dont la composition est la suivante; Ti-31,9 \% wt Al; Ti-35,5\% wt Al. Il s'avère que pour ces concentrations, l'alliage présente une solidification dite «péritectique » (Fig. 1). L'alliage commence à se solidifier en formant une phase primaire. Dès que le système atteint une certaine température (qualifiée de température péritectique); la réaction suivante se produit :

\section{Liquide + phase primaire $(\beta) \rightarrow$ phase secondaire $(\alpha)$}

Autrement dit, l'alliage au cours de la solidification va être constitué de deux phases solides distinctes. Même si la phase primaire doit normalement disparaître au cours du temps par diffusion solide-solide au profit de la phase secondaire, la coexistence des deux phases est peu souhaitable. On souhaite savoir si la convection influence ou non cette coexistence.

Le modèle numérique que l'on a utilisé prend en compte l'ensemble des aspects mentionnés.

\section{Présentation du modèle}

La grande difficulté dans la réalisation d'un modèle de solidification réside dans la grande diversité des échelles en présence. Il est impossible de négliger ce qui se passe à l'échelle microscopique, tant les phénomènes qui s'y déroulent, influencent les phénomènes macroscopiques. Idéalement, il faudrait résoudre l'ensemble des équations locales avec les conditions aux interfaces correspondantes. Malheureusement une telle résolution n'est pas envisageable. Dans cette optique, un modèle multiphasique de type Euler-Euler a été développé [2]. Les équations globales sont obtenues par un procédé de moyennage statistique d'ensemble. Comparé à la moyenne volumique qui a été largement utilisée, le moyennage statistique d'ensemble présente de nombreux avantages. Le plus important d'entre eux est de permettre le traitement rigoureux de la coexistence de différentes phases solides. 
Tableau 1. Propriétés physiques du TiAl (31,9 wt\%), Bai [5].

\begin{tabular}{lll}
\hline Propriétés thermophysiques & Symbole/unités & Valeur \\
\hline Température de liquidus & $T_{L}[\mathrm{~K}]$ & 1813,68 \\
Conductivité thermique & $\lambda\left[\mathrm{W} \cdot \mathrm{m}^{-1} \cdot \mathrm{K}^{-1}\right]$ & 23 \\
Densité & $\rho\left[\mathrm{kg} \cdot \mathrm{m}^{-3}\right]$ & 3800 \\
Chaleur latente de fusion & $L\left[\mathrm{~J} \cdot \mathrm{kg}^{-1}\right]$ & $411,5 \times 10^{3}$ \\
\hline
\end{tabular}

Les équations moyennées sont fermées par le modèle dit « modèle de la maille ». Par ailleurs, un modèle d'enveloppe est utilisé pour paramétrer les petites échelles [3,4], i.e. les échelles dendritiques.

Dans l'absolu la formulation mathématique du problème permet de prendre en compte un nombre important de phases. Pour notre étude, trois phases ont été prises en considération : la phase liquide, la phase solide primaire, la phase solide secondaire. Le modèle mathématique a été implémenté dans le logiciel Fluent ${ }^{\circledR}$.

\section{Résultats}

\subsection{Présentation du cas d'étude}

Considérons un échantillon cylindrique, de diamètre $8 \mathrm{~mm}$ et de longueur $50 \mathrm{~mm}$ composé d'un alliage de Ti-35,2 \%wt Al. Les propriétés thermophysiques sont données dans le tableau 1. Cet échantillon subit une solidification de type Bridgman : l'échantillon, soumis à un gradient de température fixe $\left(G=5000 \mathrm{~K} . \mathrm{m}^{-1}\right)$, est refroidi par le bas avec une vitesse constante de refroidissement $\left(0,5 \mathrm{~K} . \mathrm{s}^{-1}\right)$.

La modélisation est menée dans un premier temps dans un cas de microgravité puis dans un cas de convection forcée. Cette dernière nous est apparue plus intéressante que la convection naturelle. En effet, les effets hydrodynamiques sont plus prédictibles dans le cas d'une convection forcée. Pour ce faire, on soumet le lingot à un brassage électromagnétique rotatif. La force électromagnétique est donnée par l'équation (1). Elle orientée uniquement suivant l'axe du cylindre (axe $y$ ).

$$
F_{y}(r)=F_{0} \exp \left(\frac{-2\left(r-R_{\text {cylindre }}\right)}{\delta}\right)
$$

Avec $F_{0}=150 \mathrm{~N} . \mathrm{m}^{-3}, \delta=0,0013 \mathrm{~m}$ (épaisseur de peau), $R_{\text {cylindre }}=4 \mathrm{~mm}$. Cette force va créer un mouvement ascendant au centre de l'échantillon.

\subsection{Résultats en microgravité}

\subsubsection{Sur les ségrégations}

La figure 2 permet de voir l'évolution de la concentration moyenne au cours du temps pour deux abscisses différentes. La courbe bleue (losange) est associée à l'abscisse $y=0,1 \mathrm{~mm}$ et la courbe rouge (croix) à $y=1 \mathrm{~mm}$.

On constate que la concentration en aluminium en $y=0,1 \mathrm{~mm}$ diminue fortement, ce qui est logique puisque

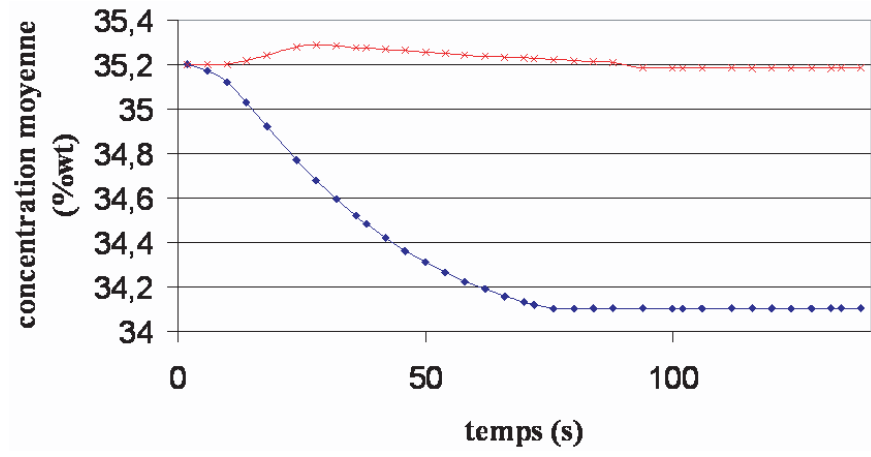

Fig. 2. Cas de microgravité. Evolution de la concentration moyenne en aluminium au cours du temps aux abscisses : $y=$ $0,1 \mathrm{~mm}$ (courbe bleue, losange) et $y=1 \mathrm{~mm}$ (courbe rouge, croix).

ce point est solidifié dès les premiers instants. En revanche, en $y=1 \mathrm{~mm}$, on observe une augmentation de la concentration suivie d'une diminution. En effet, toute la partie du lingot située à une abscisse inférieure à $1 \mathrm{~mm}$ rejette du soluté en se solidifiant, ce qui a pour conséquence d'augmenter la concentration en aluminium dans le bain liquide. Quand la partie située à $y=1 \mathrm{~mm}$ se solidifie, elle rejette à son tour le soluté et s'appauvrit en conséquence. On remarque que l'appauvrissement est beaucoup plus important pour l'abscisse $y=0,1 \mathrm{~mm}$ que pour l'abscisse $y=1 \mathrm{~mm}$. Ainsi que cela a été mentionné précédemment, une partie du lingot solidifiée dans les premiers temps a une composition plus pauvre en soluté qu'une partie solidifiée plus tardivement.

\subsubsection{Sur la distribution des phases}

Les fractions de solide primaire, de solide secondaire et de solide total sont représentées sur la figure 3. Sur chacune des figures sont également représentées la température péritectique (ligne continue) et la température de liquidus de la première phase (ligne pointillée). Les deux phases sont présentes dans l'échantillon. Néanmoins il y a une zone où seule la phase primaire est présente; il s'agit de la zone située entre la température de liquidus et la température péritectique (Fig. 3 en haut à gauche). Ceci est logique puisque la phase secondaire ne peut pas apparaître à une température supérieure la température péritectique. La zone d'apparition de la phase primaire est donc distincte de la zone d'apparition de la phase secondaire.

\subsection{Résultats en convection forcée}

\subsubsection{Influence de la convection sur les ségrégations}

La force électromagnétique appliquée au liquide a pour effet de drainer le fluide de l'extérieur vers le centre du lingot. Les distributions de soluté et de température sont influencées par ce mouvement. La figure 4 représente 

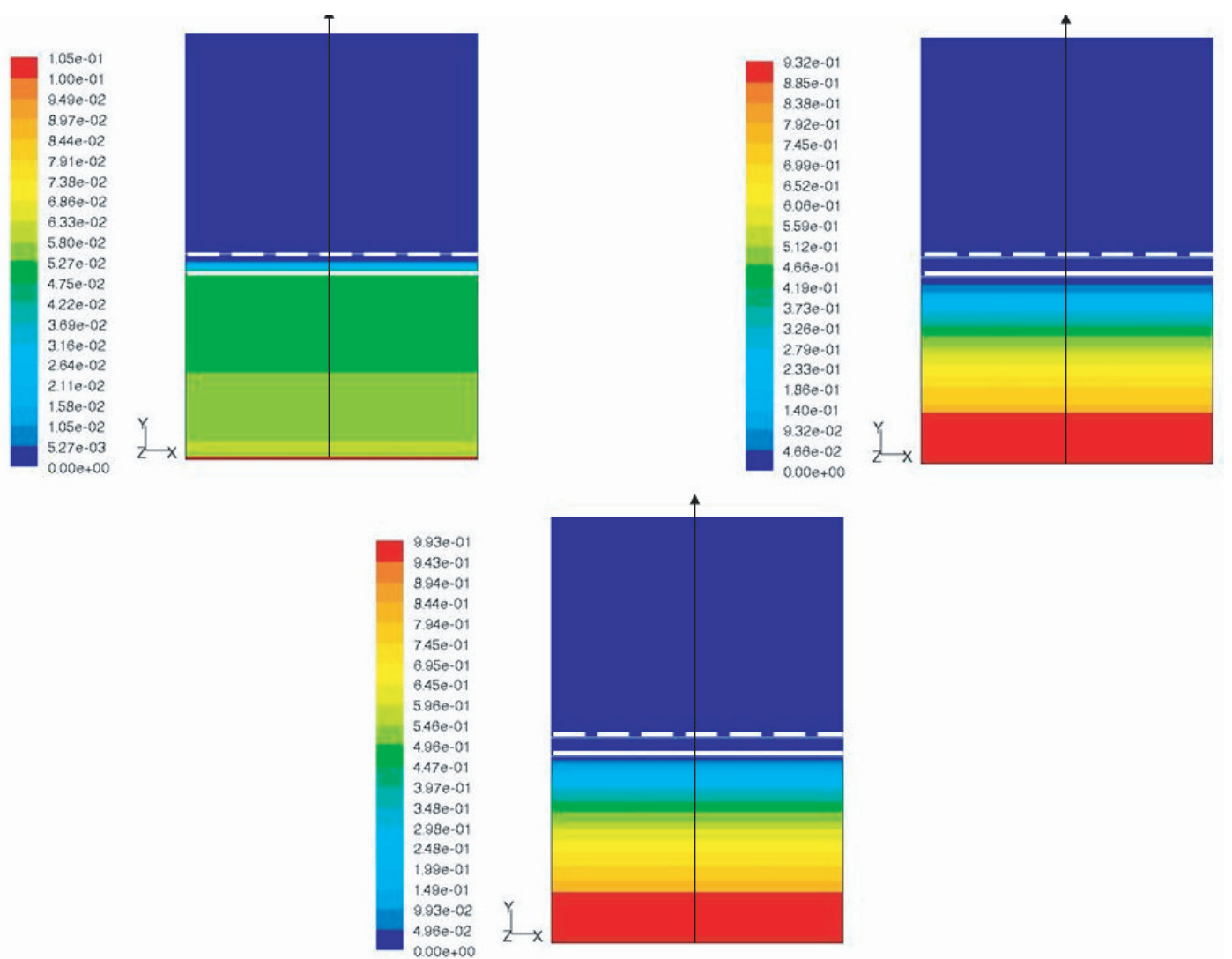

Fig. 3. Cas de microgravité. Cartes de fraction solide à $t=100 \mathrm{~s}$. En haut à gauche : fraction de solide primaire. En haut à droite : fraction de solide secondaire. En bas : fraction de solide totale.
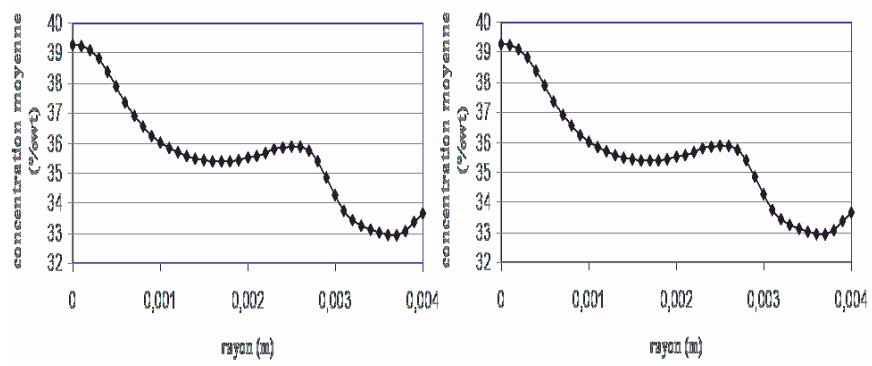

Fig. 4. Convection forcée. Évolution de la concentration moyenne le long du rayon du lingot à l'abscisse $y=0,002 \mathrm{~m}$ $t=100 \mathrm{~s}$.

l'évolution de la concentration le long du rayon à une abscisse $y=0,002 \mathrm{~m}$ et à $t=100 \mathrm{~s}$ de solidification. On observe un appauvrissement en soluté à l'extérieur et un enrichissement au centre. La répartition du soluté est directement liée au sens de l'écoulement. Or cette répartition a un impact sur la solidification. En effet, le diagramme de phase de l'alliage Ti-Al indique que plus l'alliage est enrichi en aluminium, plus la température de liquidus diminue. Étant donnée la répartition de soluté observée, les côtés du lingot vont se solidifier avant le centre. C'est effectivement observé sur la figure 5 (figure du bas) qui représente la répartition de la fraction solide au bout de $100 \mathrm{~s}$ de solidification. La fraction solide est plus importante sur les côtés que sur le centre.

\subsubsection{Influence de la convection sur les phases solides}

Les fractions de solide primaire, de solide secondaire et de solide total sont représentées sur la figure 5. Sur chacune des trois figures sont également tracées l'isotherme péritectique (ligne continue) et les courbes de liquidus (ligne pointillée) pour la phase primaire et pour la phase secondaire. On observe qu'il y a une ségrégation de phases. La phase primaire est située exclusivement sur les côtés tandis que la phase secondaire est présente aussi bien au centre qu'à l'extérieur du lingot. En effet, la phase primaire ne peut apparaître que dans un endroit où la température de liquidus est supérieure à la température péritectique; sur la figure 5 cette condition n'est réalisée que sur les côtés. L'enrichissement au centre est supérieur à la concentration péritectique. Il n'y a que la phase secondaire qui puisse germer au centre. Il faut noter que la phase secondaire est également présente sur les côtés. En effet, elle vient « compléter » la phase primaire qui s'est formée auparavant, dès que la température est inférieure ou égale à la température péritectique.

\section{Conclusions}

Le modèle numérique établi a permis de prendre en compte les phénomènes de ségrégations ainsi que la 


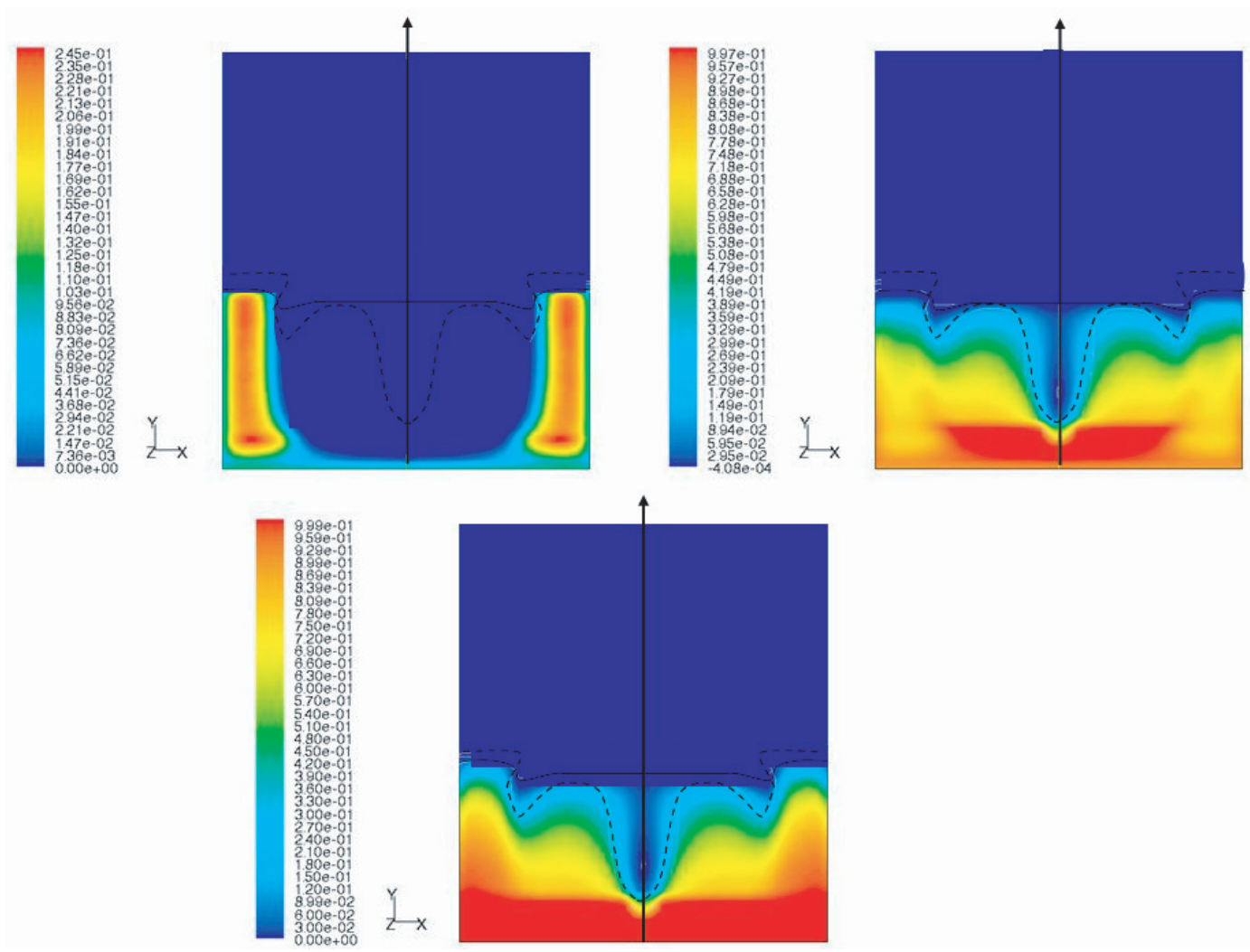

Fig. 5. Convection forcée. Cartes de fraction solide à $t=100 \mathrm{~s}$. En haut à gauche : fraction de solide primaire. En haut à droite : fraction de solide secondaire. En bas : fraction de solide totale.

solidification péritectique propre aux alliages de titanealuminium. La comparaison des résultats en microgravité et en convection forcée montrent l'importance des phénomènes convectifs sur la localisation et le devenir des ségrégations et des différentes phases. En ce qui concerne les ségrégations, la convection forcée ne permet pas de les faire disparaître mais elle en permet du moins le contrôle. En ce qui concerne la sélection de phase, la convection forcée permet également de contrôler les zones d'apparition de la phase secondaire.

Acknowledgements. This work has been funded by the followings supports: EC under PI-IMPRESS (Contract Number: NMP3-CT-2004-500635).

Remerciements. Ce travail a été realisé dans le cadre du projet IMPRESS soutenu par la commuaunté européenne : EC under PI-IMPRESS (Contrat Number: NMP3-CI-2004-500635)

\section{References}

[1] A. Hellawell, J.R. Sarazin, R.S. Steube, Channel convection in party solidified systems, Phil. Trans. R. Soc. London A 345 (1993) 507-544

[2] A. Ciobanas, Modélisation statistique de la solidification colonnaire/équiaxe des alliages binaires, INPG, Grenoble, 2006

[3] M. Rappaz, Ph. Thévoz, Solute diffusion model for the equiaxed dendritic growth, Acta Metallurgica 35 (1987) 1487-1497

[4] C.Y. Wang, C. Beckermann, A multiphase model for dendritic alloy solidification, Metallurgical and Materials Transactions A 24 (1993) 2787-2802

[5] Y. Bai, D. Xu, L. Mao, J. Guo, H. Fu, FEM/FDM-joint simulation for transport phenomena in directionally solidifying shaped TiAl casting under electromagnetic field, ISIJ Int. 44 (2004) 1173-1179 